\title{
Word use and sound change
}

\author{
NICOLAI PHARAO
}

Both usage based phonology and sociolinguistics make strong predictions about the spread of an on-going sound change through the lexicon and the speech community. For reductions, usage-based phonology has shown that such changes begin in high frequency words and spread to low frequency words. In sociolinguistic, change is charted by comparing tendencies for use of a new variant across generations, i.e. in apparent time, or across the life span, i.e. in real time. This paper investigates the connection between the two by studying the loss of $[\mathrm{w}]$ before [ð] $]$ in the speech of middle class informants from Copenhagen. The analyses show that the loss of $[\mathrm{w}]$ is correlated with word frequency in the way predicted by usage based phonology: high frequency words have a greater tendency for deletion that low frequency words. The effect is also found in the transmission of the process from the older generation to the younger generation: younger speakers have a greater tendency for [w]-loss in mid and low frequency words than older speakers do. The pattern is also found at the level of individual speakers. Finally, some evidence is also found for the frequency effect within speakers across the lifespan, as those speakers who are seen to increase their use of forms without $[\mathrm{w}]$ also tend to follow the path of frequency. It is argued that these results support an exemplar based model of the mental lexicon where frequency of use is incorporated into representations of word forms. 